\title{
EDITORIAL
}

\section{Advances in Management of Chronic Hepatitis C infection}

Hepatitis C Virus (HCV) infection poses a huge burden worldwide, as well as on the Bangladeshis, being a leading cause of all forms of chronic liver diseases next only to $\mathrm{HBV}^{1,2}$. A recent study in Bangladesh showed a prevalence of $0.88 \%$, though figures vary due to lack of representative population study in Bangladesh ${ }^{2}$. The advent of new generation oral antiviral therapy has led to major improvements in efficacy and tolerability but has also resulted in an explosion of data with increased treatment choice complexity. The questions of who should be treated and with what regimen, will be increasingly complex to address and will require careful consideration. As therapy improves, identification and care of patients who need treatment will be the next challenge ${ }^{3}$. We need to update ourselves continuously to provide optimum treatment to our patients.

$\mathrm{HCV}$ is a positive-strand RNA virus encoding a polyprotein that undergoes proteolytic cleavage to 10 polypeptides, each with distinct functions. The structural proteins consist of two envelope glycoproteins, both of which are targets of host antibody response, and the core protein, which interacts with progeny viral genomes for assembly of the virus. The nonstructural proteins NS2, NS3, NS4A, NS4B, NS5A, and NS5B form a complex with viral RNA to initiate viral replication in a cytoplasmic membranous structure. The new direct-acting antiviral agents (DAAs) target these non-structural proteins to prevent viral replication ${ }^{4}$.

AASLD/IDSA recommendations (http://www. hcvguidelines.org) for One-time HCV Testing:

One-time HCV testing is recommended for persons born between 1945 and 1965, without prior ascertainment of risk. Other persons should be screened for risk factors for HCV infection, and one-time testing should be performed for all persons with behaviors, exposures, and conditions associated with an increased risk of $\mathrm{HCV}$ infection.

\section{Risk behaviors}

1. Injection-drug use (current or ever, including those who injected once)

2. Intranasal illicit drug use
Risk exposures

1. Persons on long-term hemodialysis (ever)

2. Persons with percutaneous/parenteral exposures in an unregulated setting

3. Healthcare, emergency medical, and public safety workers after needle sticks, sharps, or mucosal exposures to $\mathrm{HCV}$-infected blood

4. Children born to HCV-infected women

5. Prior recipients of transfusions or organ transplants, including persons who:

Were notified that they received blood from a donor who later tested positive for $\mathrm{HCV}$

Received a transfusion of blood or blood components, or underwent an organ transplant before July 1992

6. Received clotting factor concentrates produced before 1987

7. Persons who were ever incarcerated

Other considerations:

HIV infection

Sexually active persons about to start pre-exposure prophylaxis (PreP) for HIV

Unexplained chronic liver disease and/or chronic hepatitis including elevated alanine, aminotransferase levels

Solid organ donors (deceased and living)

Treatment guidelines are constantly evolving due to emerging regimens and real world treatment data. It is recommended to treat for all patients with chronic $\mathrm{HCV}$ infection, except those with short life expectancies that cannot be remediated by treating $\mathrm{HCV}$, by transplantation, or by other directed therapy. The goal of treatment of HCV-infected persons is to reduce allcause mortality and liver related health adverse consequences, including end-stage liver disease and hepatocellular carcinoma, by the achievement of virologic cure as evidenced by a sustained virologic 
response, defined as undetectable HCV RNA in the blood after the end of HCV treatment, either at 12 weeks (SVR12) or at 24 weeks (SVR24). Special population like HIV co-infection, renal impairment etc. requires additional care.

Boceprevir and Telaperevir were the first generation protease inhibitors followed by Sofosbuvir (SOF), Simeprivir (SIM), approved by the Food and Drug Administration (FDA) in 2013. This was followed by the Ledipasvir-Sofosbuvir (LDV/SOF) combination and Ombitasvir-paritaprevir-ritonavir-dasabuvir combination (3D regimen). Following, Daclatasvir and Ombitasvir-paritaprevir-ritonavir combination (2D regimen) were approved by the FDA for the treatment of genotype 3 and 4 respectively. Most recently, ElbasvirGrazoprevir (EBV/GZR) was FDA approved for the treatment of genotypes 1 and 4 . The choice and duration of treatment will be dependent on several key factors including genotype, treatment experience, presence of cirrhosis, cost, potential drug interactions, and identification of special populations (end-stage renal disease, post-liver transplant, HIV co-infection) ${ }^{3}$.

WHO has updated its guidelines for the screening, care and treatment of persons with chronic hepatitis $\mathrm{C}$ infection in April 2016 (http://www.who.int/hepatitis/ publications/hepatitis-c-guidelines-2016/en/). The guideline strongly recommends that DAA regimens be used for the treatment of persons with hepatitis $\mathrm{C}$ infection rather than regimens with pegylated interferon and ribavirin. Though for patients with HCV genotype 3 infection with cirrhosis and patients with genotypes 5 and 6 infection with and without cirrhosis, an interferonbased regimen: sofosbuvir/pegylated interferon and ribavirin is still recommended as an alternative treatment option. The use of boceprevir- or telaprevir-containing regimens is no longer recommended. Summary of recommended preferred regimens with treatment durations is as follows:

\section{Persons without cirrhosis:}

\begin{tabular}{|c|c|c|c|}
\hline & Daclatasvir/ sofosbuvir & Ledipasvir/ sofosbuvir & Sofosbuvir/ ribavirin \\
\hline Genotype 1 & 12 weeks & 12 weeks & \\
\hline Genotype 2 & & & 12 weeks \\
\hline Genotype 3 & 12 weeks & & 24 weeks \\
\hline Genotype 4 & 12 weeks & 12 weeks & \\
\hline Genotype 5 & & 12 weeks & \\
\hline Genotype 6 & & 12 weeks & \\
\hline
\end{tabular}

\section{Persons without cirrhosis}

\begin{tabular}{|c|c|c|c|c|c|}
\hline & $\begin{array}{l}\text { Daclatasvir/ } \\
\text { sofosbuvir }\end{array}$ & $\begin{array}{l}\text { Daclatasvir/ } \\
\text { sofosbuvir } \\
\text { ribavirin }\end{array}$ & $\begin{array}{l}\text { Ledipasvir/ } \\
\text { sofosbuvir }\end{array}$ & $\begin{array}{l}\text { Ledipasvir/ } \\
\text { sofosbuvir/ } \\
\text { ribavirin }\end{array}$ & $\begin{array}{c}\text { Sofosbuvir/ } \\
\text { ribavirin }\end{array}$ \\
\hline Genotype 1 & 24 weeks & 12 weeks & 24 weeks & 12 weeks* & \\
\hline Genotype 2 & & & & & 16 weeks \\
\hline Genotype 3 & & 24 weeks & & & \\
\hline Genotype 4 & 24 weeks & 12 weeks & 24 weeks & 12 weeks* & \\
\hline Genotype 5 & & & 24 weeks & 12 weeks* & \\
\hline Genotype 6 & & & 24 weeks & 12 weeks* & \\
\hline
\end{tabular}

*If platelet count $<75 \times 103 / \mathrm{iL}$, then 24 weeks' treatment with ribavirin should be given 
Genotype (GT) 1 has the most treatment options and physicians should choose as per clinical situations. The only approved oral regimen for GT 2 is SOF with RBV for $12-16$ weeks. Genotype 3 is prevalent in Bangladesh ${ }^{5}$ and currently it is the one most difficult to treat. Although the reasons are unclear, GT3 patients tend to have more risk factors associated with treatment resistance. GT3 is associated with a higher risk of developing cirrhosis $(\mathrm{HR}=1.4,95 \% \mathrm{CI},[1.32-1.5])$ and hepatocellular carcinoma ( $\mathrm{HR}=1.66,95 \% \mathrm{CI},[1.48-1.85])$. However, treatment naïve, non-cirrhotic, HCV GT3 infected patients still tend to have excellent response to DAA therapy. Currently, addition of PEG-IFN to SOF with RBV for 12 weeks is the primary AASLD/IDSA recommended therapy for GT3 treatment experienced with cirrhosis. Most recently, EBV/GZR was FDA approved for the treatment of GT4 infection for 12 weeks in treatment naïve patients and 16 weeks with the addition of RBV in treatment experienced patients ${ }^{3}$.

Conclusion: Chronic hepatitis $\mathrm{C}$ treatment will continue to evolve with next generation regimens. The goals of future therapy will include 1) Pan-genotypic activity with high barrier to resistance 2) Simplified dosing 3)
RBV free and 4) Shorter duration of treatment. The future regimens will fill the gaps.

(J Bangladesh Coll Phys Surg 2017; 35: 48-50)

\section{Khan Abul Kalam Azad}

Professor of Medicine, Dhaka Medical College

Syed Ghulam Mogni Mowla

Assistant Professor of Medicine, Dhaka Medical College

\section{Reference:}

1. B. L. Pearlman. Protease inhibitors for the treatment of chronic hepatitis $\mathrm{C}$ genotype- 1 infection: the new standard of care. The Lancet Infectious Diseases 2012; 12(9): 717-728.

2. Mahtab MA, Rahman S, Karim F, et al. Epidemiology of Hepatitis C Virus in Bangladeshi General Population. BSMMU J 2009; 2(1): 14-17

3. Zhang J, Nguyen D, and Hu KQ. Chronic Hepatitis C Virus Infection: A Review of Current Direct Acting Antiviral Treatment Strategies. N Am J Med Sci (Boston). 2016 April ; 9(2): 47-54.

4. Liang TJ and Ghany MG. Current and Future Therapies for Hepatitis C Virus Infection. $N$ Engl J Med 2013;368:1907-17

5. Islam MS1, Miah MR, Roy PK, et al. Genotypes of hepatitis $\mathrm{C}$ virus infection in Bangladeshi population. Mymensingh Med J. 2015 Jan;24(1):143-51. 